\title{
Análisis de biomecánica digital en marcha protésica de paciente con amputación por encima de rodilla
}

\author{
Cristian Camilo Benavidez Noguera', Andrea Torres Ruiz ${ }^{1}$ \\ 1.Grupo de Instrumentación y Rehabilitación Biomédica GIRB. \\ Universidad Manuela Beltrán, Bogotá, Colombia. \\ Correspondencia: andrea.torres@umb.edu.co \\ Recibido: 16-03-09 / Aceptado: 18-05-09
}

\begin{abstract}
Resumen
Esta investigación, que se realizó en el Laboratorio de Biomecánica Digital BIOMED, muestra un estudio de caso de un paciente con amputación por encima de la rodilla y el mecanismo de rodilla monocéntrica, el cual emplea sistemas optoelectrónicos para capturar los movimientos y placas transductoras de fuerza, con el fin de obtener datos de cinética y cinemática de la extremidad. Los resultados fueron analizados para tratar de reconocer los patrones más importantes, las características y posibles mejoras en la marcha, lo cual podría proporcionar algunas bases para el futuro diseño de prótesis, de tal forma que sean cada vez más funcionales y similares de acuerdo al patrón de la marcha normal.

Palabras clave: amputación, análisis digital, biomecánica, prótesis.
\end{abstract}

\section{Abstract
Biomechanical Analysis of Ongoing Digital Prosthetic Amputee above Knee}

This research, conducted at the BIOMED Digital Biomechanics Laboratory, shows a case study of a patient with amputation above the knee and the monocentric knee mechanism, which employs optoelectronic systems to capture the movement and force transducers plates to obtain data on kinetics and kinematics of the limb. The results were analyzed to try to recognize the major patterns, characteristics and possible improvements in gait, which could provide some basis for the future design of artificial limbs, so they are increasingly functional and similar to the pattern of normal gait.

Keywords: amputation, biomechanics, digital analysis, prosthesis. 


\section{Introducción}

En la actualidad es posible encontrar diversas investigaciones acerca del análisis de marcha normal y patológica, sin embargo, no hay suficiente información sobre la marcha protésica y sus diferentes modelos $(1,2)$. Se realizó el estudio de la marcha protésica transfemoral, con mecanismo de rodilla monocéntrica, a través del uso equipos del Laboratorio de Biomecánica Digital BIOMED de la Universidad Manuela Beltrán, con el fin de observar e interpretar el patrón que presenta la marcha con el uso de éste tipo de prótesis que se encuentra en el mercado y poder argumentar posibles mejoras en los diseños de prótesis, que permitan a los pacientes llevar una marcha más cómoda y confiable, lo cual favorece una mejor calidad de vida, sin ningún tipo de complejo o dificultad en su vida diaria.

\section{Materiales y métodos}

Para la caracterización de la marcha protésica y su respectivo análisis, se tuvo como principal herramienta, los equipos del laboratorio de Biomecánica Digital BIOMED de la Universidad Manuela Beltrán.

\section{Equipos y software}

Para valorar la marcha, el laboratorio de biomecánica cuenta con seis cámaras optoelectrónicas de alta resolución, encargadas de registrar los marcadores que se ubican en la superficie corporal del paciente, con una frecuencia de adquisición de 100/120 Hz. También hay dos cámaras de video digital que monitorean el movimiento del paciente en diferentes planos. Para el registro cinético se contó con dos placas de fuerza multiaxial, encargadas de medir la fuerza de reacción para suministrar la información pertinente a los momentos externos de las articulaciones de las extremidades inferiores.

El laboratorio cuenta con el software Biomech ${ }^{\oplus}$, que es el encargado de la digitalización de la información del movimiento de los arcadores en el espacio y en el tiempo, el software Tracklab $^{\oplus}$, permite visualizar la imagen en el espacio, las curvas de posición en $\mathrm{x}, \mathrm{y}$ y z, la aceleración y la velocidad de cada uno de los marcadores ubicados en el paciente. También permite crear un archivo para visualizar el modelo biomecánico. El software SmartAnalyzer ${ }^{\circ}$, que realiza los cálculos espaciales de los puntos de referencia sobre el modelo biomecánico, visualiza el resultado de la cinética y cinemática del movimiento en un espacio 3D. Se usó el software Elite Clinic ${ }^{\oplus}$, que integra el análisis cinético y cinemático, para realizar finalmente la descripción del ciclo de la marcha.

\section{Muestra}

El estudio se llevó a cabo en un paciente de 36 ańos de edad, 64 kilos de peso y $1.79 \mathrm{~m}$ de altura, víctima del conflicto armado que vive el país, con amputación en la pierna derecha por encima de la rodilla. La prótesis pesa 3.5 kilogramos. Para realizar el análisis computarizado de marcha fue necesario tomar las medidas antropométricas del paciente, tanto en el miembro sano como en el miembro con la prótesis. Luego, se colocaron los marcadores reflectivos en el cuerpo del paciente de acuerdo al protocolo Davis, que hace uso de 22 marcadores, que son registrados por las cámaras. Por medio del procesamiento de las señales, se realiza una reconstrucción 3D de la marcha, que se ve resumida en un reporte clínico que contiene los datos personales del paciente y una serie de Figuras cinemáticas de cada articulación en diferentes planos.

Se analizaron las curvas del reporte que se obtuvo al capturar un ciclo de marcha y se relacionó cada una de éstas con las fases de la marcha, que se puede dividir en una fase de apoyo, cuyo valor normal esta en $60 \%$ del ciclo de la marcha y una fase de oscilación de 40\% (3-5), como se aprecia en la Figura 1. Se observó detalladamente el movimiento articular (comportamiento de los ángulos) en los diferentes planos biomecánicos (sagital, frontal y coronal) (6), ya que el movimiento coordinado en tobillo, pie, rodilla y cadera, son esenciales para el desarrollo una buena locomoción $(7,8)$. Además, se analizó e identificó la cinética de las articulaciones con respecto a los momentos externos de cada articulación a lo largo de la marcha y cómo éstos son el producto de las diferentes fuerzas internas generadas por los músculos, ligamentos, tendones y fuerzas de contacto, Figura 1.

Tanto la fase de apoyo y de oscilación se dividen en subfases, como se observa en la Tabla1.

Durante el apoyo se encuentra el CI con un valor de 0-2\% del porcentaje total del ciclo de marcha, el AI con 0-10\%, el AM con 10-30\%, el AF con 30-50\%, la OP con 50-60\%; durante la oscilación se encuentra la OI con 60- 


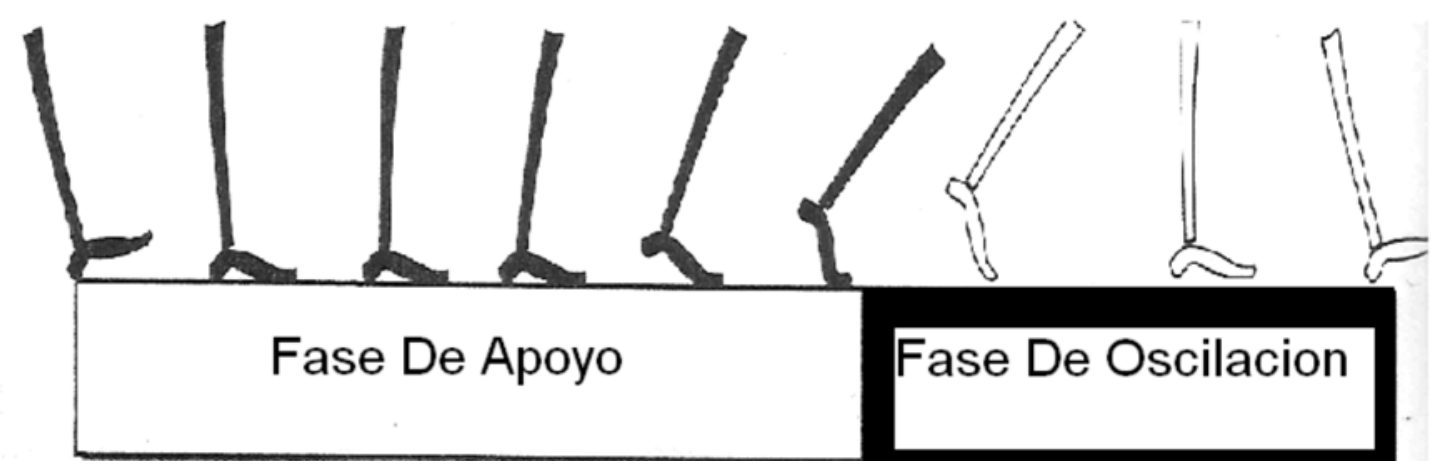

Figura 1. Fases de la marcha humana normal. PERRY, Jacquelin, GAIT ANALYSIS, Normal and Pathological Function. NJ: Slack Incorporated, 1992. p. 4.

Tabla 1. Cinemática de las articulaciones de cadera, rodilla y tobillo a lo largo de las fases de la marcha.

\begin{tabular}{|c|c|c|c|c|c|c|c|c|}
\hline $\begin{array}{l}\text { Articulación } \\
\text { Fase }\end{array}$ & $\mathrm{Cl}$ & Al & AM & AF & OP & 이 & OM & OF \\
\hline Cadera & Flexión & Flexión & Extensión & Extensión & $\begin{array}{l}\text { Reduce } \\
\text { extensión }\end{array}$ & Flexión & Flexión & Flexión \\
\hline Rodilla & Extensión & Flexión & $\begin{array}{c}\text { Finaliza flexión } \\
\text { y comienza } \\
\text { extensión }\end{array}$ & $\begin{array}{l}\text { Termina } \\
\text { extensión y } \\
\text { comienza } \\
\text { flexión }\end{array}$ & Flexión & Flexión & $\begin{array}{c}\text { Flexión hacia } \\
\text { extensión }\end{array}$ & $\begin{array}{l}\text { Flexión } \\
\text { hacia } \\
\text { extensión }\end{array}$ \\
\hline Tobillo & Neutro & $\begin{array}{l}\text { Flexión } \\
\text { plantar }\end{array}$ & $\begin{array}{l}\text { Dorsiflexión } \\
\text { controlada }\end{array}$ & $\begin{array}{l}\text { Flexión } \\
\text { plantar }\end{array}$ & $\begin{array}{l}\text { Flexión } \\
\text { plantar }\end{array}$ & $\begin{array}{l}\text { Dorsi } \\
\text { flexión }\end{array}$ & $\begin{array}{l}\text { Dorsi flexión } \\
\text { hacia } 0^{\circ}\end{array}$ & $\begin{array}{l}\text { Cercano a } \\
\text { la neutra } 0^{\circ}\end{array}$ \\
\hline
\end{tabular}

$\mathrm{Cl}=$ Contacto inicial, $\mathrm{Al}=$ Apoyo inicial, $\mathrm{AM}=$ Apoyo medio, $\mathrm{AF}=$ Apoyo final, $\mathrm{OP}=$ Fase previa de oscilación, $\mathrm{Ol}=$ Oscilación inicial, $\mathrm{OF}=\mathrm{Oscilación} \mathrm{final.}$

$73 \%$, la OM con $73-87 \%$ y la OF con $87-100 \%(7,8)$. En estas fases cada articulación presenta un comportamiento cinemático que ha sido analizado y determinado a lo largo de los años por diferentes autores con el fin de establecer un patrón característico (9-11).

\section{Resultados}

Con este estudio se obtuvo un reporte clínico conformado por una serie de datos que se organizaron en una serie de tablas y Figuras. En la Tabla 2 se muestran los parámetros temporales para cada miembro y los valores de referencia para cada patrón con sus respectivos límites positivos y negativos. Para el miembro derecho, que tiene la prótesis (AK), los datos son mostrados con asterisco.

Durante la fase de apoyo el valor que tiene el miembro que lleva la prótesis, está por debajo del valor de referencia. Esto quiere decir que el paciente a la hora de apoyarse sobre ésta, intenta hacerlo de una forma rápida, ya que el control que se ejerce no es el mismo al de un paciente normal ni al de su miembro sano, que es regulado por el sistema neuromuscular. Debido a esta falta de control autónomo sobre la prótesis, el paciente presenta cierto

Tabla 2. Parámetros temporales

\begin{tabular}{ccccc|} 
& \multicolumn{2}{c}{ Parámetros temporales } & \multicolumn{2}{c|}{ Valores de referencia } \\
& RT $^{*}$ & LT & RT $^{*}$ & LT \\
\hline Fase apoyo [\%] & 58 & 62,4 & $59,6 \pm 1,2$ & $59,3 \pm 1,8$ \\
Fase de balanceo [\%] & 42 & 37,6 & $40,4 \pm 1,2$ & $40,7 \pm 1,8$ \\
\hline Tiempo doble apoyo [\%] & 9,2 & 11,8 & $13,4 \pm 1,1$ & $8,3 \pm 0,6$ \\
\hline Tiempo fase apoyo [s] & 0,69 & 0,715 & $0,63 \pm 0,21$ & $0,626 \pm 0,425$ \\
\hline Tiempo fase balanceo [s] & 0,5 & 0,43 & $0,426 \pm 0,16$ & $0,429 \pm 0,23$ \\
Tiempo zancada [s] & 1,19 & 1,145 & $1,056 \pm 0,26$ & $1,055 \pm 0,52$ \\
\hline
\end{tabular}

Cadencia [pasos/minuto]

[hz]

103,516

$113,845 \pm 4,305$

$\mathrm{RT}=$ Pierna derecha, $\mathrm{LT}=$ Pierna izquierda. 
Tabla 3. Parámetros de distancia

\begin{tabular}{ccccc} 
& \multicolumn{2}{c}{ Parámetros de distancia } & \multicolumn{2}{c|}{ Valores de referencia } \\
& RT $^{*}$ & LT & RT $^{*}$ & LT \\
\hline Longitud de paso $(\mathrm{m})$ & 0,649 & 0,607 & $0,619 \pm 0,004$ & $0,74 \pm 0,019$ \\
Velocidad $(\mathrm{m} / \mathrm{s})$ & 1,095 & 1,121 & $1,33 \pm 0,062$ & $1,331 \pm 0,066$ \\
Velocidad balanceo $(\mathrm{m} / \mathrm{s})$ & 2,606 & 2,995 & $3,296 \pm 0,137$ & $3,275 \pm 0,184$ \\
Longitud zancada $(\mathrm{m})$ & 1,303 & 1,285 & $1,404 \pm 0,074$ & $1,402 \pm 0,06$ \\
Ancho paso $(\mathrm{m})$ & 0,188 & 0,179 & $0,11 \pm 0,026$ & $0,128 \pm 0,011$ \\
Velocidad promedio $(\mathrm{m} / \mathrm{s})$ & 1,112 & & $1,33 \pm 0,064$ & \\
\hline
\end{tabular}

$\mathrm{RT}=$ Pierna derecha, $\mathrm{LT}=$ pierna izquierda.

grado de inseguridad al asentar su prótesis. De la misma manera el tiempo de apoyo de la prótesis es muy corto, de $0.69 \mathrm{~s}$, mientras que la pierna izquierda tiene un tiempo de apoyo $0.71 \mathrm{~s}$. En la fase de balanceo se confirmó lo anterior, ya que la prótesis tiene una mayor duración respecto al miembro izquierdo, manteniéndose mayor tiempo en el aire. Finalmente la cadencia del paciente será menor al valor de referencia, retardando el ciclo de la marcha (11).

En la Tabla 3 se anotan los parámetros de distancia. La longitud de paso y de zancada del miembro derecho protésico es mayor al valor para el miembro izquierdo, esto se debe a que la prótesis es un poco larga, aproximadamente $3 \mathrm{~cm}$ mayor al miembro normal, por lo que al dar el paso derecho, el paciente muestra un movimiento de marcha hemipléjica (circonducción) que permite un mayor avance del miembro derecho. El paso izquierdo y la longitud de zancada son menores a los valores de referencia mínimos, esta diferencia se debe a que la longitud del paso izquierdo se mide mientras el miembro derecho protésico está apoyado y como el tiempo de apoyo sobre la prótesis es muy corto, la pierna izquierda no alcanza a recorrer una distancia significativa durante su fase de oscilación, sino que la oscilación es corta buscando el próximo apoyo sobre el miembro izquierdo.

La velocidad del miembro protésico es menor a la velocidad del miembro izquierdo (11). Esto se debe a que, aunque la longitud de paso del miembro derecho es mayor como consecuencia de la pronunciada longitud del miembro protésico, el tiempo empleado en recorrer dicha distancia también es mayor. Este tiempo extenso se debe al tiempo de apoyo del miembro izquierdo, que siempre será mayor al tiempo de apoyo derecho. Así mismo, se puede indicar que la velocidad de balanceo izquierdo es mayor, ya que el paciente busca siempre de forma inmediata el apoyo del miembro izquierdo, antes que tener apoyo sobre el miembro protésico, Tabla 3.

El reporte también incluyó graficas de cinemática y de cinética de las articulaciones de cadera, rodilla y tobillo. En las figuras de cinemática se analizó el comportamiento de las articulaciones a través de los movimientos de flexión y de extensión de cada una de ellas. Para la articulación del tobillo se analizó la flexión plantar y la flexión dorsal del mismo. En las figuras de cinética se analizó el momento externo de cada articulación y cómo este puede ser dorsal o frontal a lo largo de la marcha. También se obtuvieron y analizaron las figuras de potencia, en donde las trayectorias indicaron si había una generación de potencia o una absorción de potencia con su respectiva magnitud. De las graficas de cinemática, sólo se analizará la curva de la cinemática del tobillo, pues es la articulación que presenta mayor diferencia del patrón normal, Figura 2.

En la Figura 2, se puede observar como el miembro protésico presenta una flexión plantar muy acentuada en la fase de contacto inicial, esto se debe a que la parte de la prótesis que reemplaza el pie, al hacer el contacto inicial, no se realiza un contacto de forma gradual empezando por el talón, sino que el contacto inicial se da casi con toda la planta del pie de la prótesis de forma inmediata. Por eso, tras el contacto inicial, no comienza una trayectoria hacia una flexión dorsal sino que alcanza una flexión plantar de hasta $5^{\circ}$. Esto se realiza debido a que la prótesis es un elemento sólido que se mueve bajo su inercia y momento de inercia y no tiene el control de ligamentos y músculos que amortiguan de forma continua, presentando así el impacto del pie protésico de forma directa hacia una flexión 


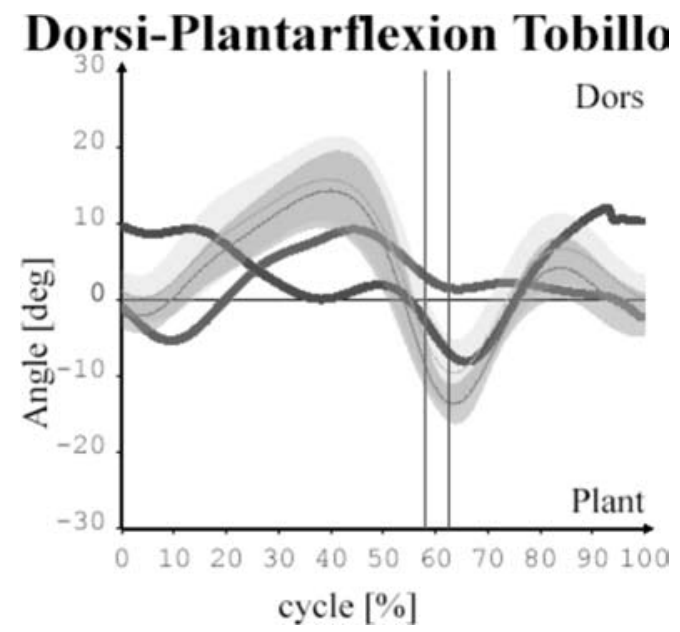

Figura 2. Cinemática del tobillo.

plantar. Ésta se mantiene ya que el pie conserva una posición horizontal inmóvil y quien se encarga de la disminución del ángulo de flexión plantar es el segmento protésico de la pierna con respecto al pie, ya que se mueve en dirección de la progresión del movimiento, a diferencia del pie protésico que permanece inmóvil durante el apoyo.

Una vez comienza la fase final del apoyo, con el despegue del talón, se puede observar una trayectoria hacia la flexión dorsal hasta alcanzar el pico más alto de flexión, para luego dirigirse de nuevo hacia la flexión plantar en la fase previa de oscilación. Sin embargo, el paciente no presenta esta flexión plantar, ya que durante la oscilación el pie protésico tiende a mantener su ángulo, casi constante debido a su naturaleza inerte y no tiene control de regulación de ligamentos y músculos, que son los encargados de llevar a cabo esta flexión dorsal-plantar durante la oscilación. Esta pequeña flexión se mantiene a lo largo de la oscilación con un valor casi constante.

En el miembro normal, en este caso el miembro izquierdo, se observa una acentuada flexión dorsal durante el contacto inicial. Esta flexión dorsal, de $10^{\circ}$ aproximadamente, se da debido a que durante el primer apoyo bipodal se presenta en el contacto inicial. El peso del cuerpo no se reparte de forma simétrica en los dos miembros sino que la mayor parte del peso recae sobre el miembro izquierdo y por eso se da esta flexión dorsal, no sólo en el pie sino que también se puede ver en la rodilla. La flexión dorsal comienza a disminuir al mismo tiempo que transcurre la fase de apoyo y llega a cero una vez se da el correcto posicionamiento del pie izquierdo. Durante la oscilación, el miembro izquierdo presenta la flexión plantar normal para luego flexionarse de forma dorsal, sin embargo en la fase final de la oscilación no presenta una flexión dorsal normal, sino que se da una flexión dorsal alta de hasta $10^{\circ}$, esto se debe al afán del paciente de apoyarse de nuevo sobre su miembro izquierdo.

\section{Discusión}

Al hablar de la marcha protésica transfemoral, se deben tener en cuenta varios aspectos y no sólo enfocarse en el diseño de la prótesis o su mecanismo de rodilla. Debido al gran daño biomecánico por la pérdida de importantes estructuras tras la amputación, son varios los factores que influyen durante la marcha y todos de especial atención (7). La marcha protésica transfemoral se obtiene tras una solución integral, son varios aspectos a trabajar si se quiere llegar a resultados parecidos a los considerados normales o que guarden un patrón parecido.

Es necesario decir que la voluntad del paciente ante la dificultad, las ganas de sobre llevarlas y tener confianza sobre su prótesis, es de suma importancia. En este estudio se trabajó con una persona dedicada a realizar actividades fuera de lo normal entre los amputados, como ascenso de pendientes y actividades que demandan gran fuerza física y emocional. Es gracias al convencimiento y disposición del paciente que es más soportable el proceso de recuperación y la consecución de las metas personales. La prótesis es un factor importante, hoy en día hay una gran variedad de diseños, pero como se ha mencionado, ésta debe verse como un diseño integral.

El encaje es una pieza fundamental, ya que de acuerdo a la fijación, se tendrá una marcha cómoda, sin dolor ni alteraciones en los planos (11). En el presente estudio el encaje y su fijación están basados en una media de silicona, con clavija distal de agarre al encaje. Este tipo de mecanismo dió gran seguridad y fijación a la prótesis, sin presentar dolor, ni gestos anormales pronunciados. El mecanismo de rodilla monocéntrica es una gran opción para cumplir sus funciones en la marcha, donde el punto decisivo es el mecanismo de fricción, el cual permite diferenciar la fase de apoyo de la de oscilación.

Un mecanismo de fricción hidráulico es excelente, ya que cumple su función de extensión en la fase de apoyo y su de flexión durante la oscilación, soportando 
varias velocidades sin precipitar al paciente a una caída o problemas de estabilidad. El tobillo protésico puede ser más funcional, tendiendo a asemejarse al comportamiento biomecánico del tobillo normal. En este caso el tobillo cumplía su fase de flexión dorsal y flexión plantar, y a través del diseño se tenía un mecanismo que fijaba los limites de las flexiones pero no se contaba con un mecanismo de regulación de las mismas.

Los gestos de la cadera y rodilla en el campo cinemático guardan un patrón semejante al normal, pero tienen una variación mínima en magnitud y algunas veces en dirección. Las consecuencias se pueden ver en la cinética, donde los momentos se ven claramente afectados, aunque el patrón de marcha puede calificarse como muy semejante al normal.

En tobillo es donde se evidencia la mayor diferencia respecto a los valores normales, pues no se tiene un control de fricción como en el caso de la rodilla, sino que simplemente se establecen unos límites de flexión y extensión, en donde la norma es el todo o nada. Si se flexiona, se dará una flexión dorsal máxima o una flexión plantar máxima. Como resultado, se podría diseñar un modelo de tobillo que permita un movimiento regulado a través de la determinación de un umbral de peso como consecuencia del peso soportado a través de las fases por parte del tobillo.

Lograr un modelo de caracterización de la marcha protésica transfemoral $(\mathrm{AK})$ y del mecanismo de rodilla monocéntrica de manera estándar, es algo muy complejo, ya que a diferencia de la caracterización de la marcha humana normal, existen un sin número de variables, que harán de cada paciente un caso particular. Sin embargo, es posible reconocer las variables que tendrán mayor influencia en la marcha protésica y a su vez, las consecuencias más notables sobre la misma.

\section{Agradecimientos}

Al Batallón de Sanidad Militar del Ejército de Colombia por abrirnos sus puertas, escucharnos, creer en nosotros y permitir trabajar en conjunto. Al paciente, a quien se respetara su identidad, por su paciencia, disponibilidad y ser ejemplo de vida. $\mathbf{N}$

\section{Referencias}

1. Bateni H, Olney SJ. Kinematic and kinetic Variations of Below-Knee Amputee Gait. Prosthet Orthot Int. 2002;1:2-10.

2. Piaza J, Delp S. Three Dimensional Dynamic Simulation of Total Knee Replacement Motion during a Step-Up Task. J Biomech Eng. 2001;123:599-606.

3. Perry J. Gait analysis: Normal and Pathological Function. NJ: Slack Incorporated 1992;4-20.

4. Trew M, Evertt T. Fundamentos del movimiento Humano. Ed. 5, Barcelona Masson; Capítulo:Biomecánica del movimiento humano. 2006.

5. Fucci S. Biomecánica del aparato locomotor aplicada al acondicionamiento muscular. Ed. IV, Madrid Elsevier. 2003.

6. Ozkaya Nihat. Fundamentals of Biomechanics. Equilibrium, motion and deformation. Ed. 2. Springer-Verlag. 1999;20-40.

7. Sánchez J. Biomecánica de la marcha humana normal y patológica. Ed. Instituto de biomecánica de Valencia. 1999;253.

8. Raimondi P. Cinesiología y psicomotricidad: modelo psicomotor, análisis del movimiento, morfotipología humana. Ed. Pardiotribo, Barcelona. Capítulos 3 y 6.1999 .

9. Campione E, Véronis J. A statistical study of pitch target points in five languages. In: Proc. of the 5th International Conference on Spoken Language Processing, Prosody and Emotion 5. 1998.

10. Ronald Valmassi. Clinical biomechanics of the lower extremities. New York Mosby. Capítulos 1-2.1996.

11. Burstein A, Wright T. Fundamentals of orthopaedic biomechanics. Ed. Baltimore, London, Williams y Wilkins, 1994. 